\title{
Distribution and variability of esophageal eosinophilia in patients undergoing upper endoscopy
}

Evan S Dellon ${ }^{1,2}$, Olga Speck ${ }^{3}$, Kimberly Woodward ${ }^{3}$, Shannon Covey ${ }^{3}$, Spencer Rusin ${ }^{3}$, Nicholas J Shaheen ${ }^{1,2}$ and John T Woosley ${ }^{3}$

${ }^{1}$ Center for Esophageal Diseases and Swallowing, Division of Gastroenterology and Hepatology, Department of Medicine, University of North Carolina School of Medicine, Chapel Hill, NC, USA; ${ }^{2}$ Center for

Gastrointestinal Biology and Disease, Division of Gastroenterology and Hepatology, Department of Medicine, University of North Carolina School of Medicine, Chapel Hill, NC, USA and ${ }^{3}$ Department of Pathology and Laboratory Medicine, University of North Carolina School of Medicine, Chapel Hill, NC, USA

The variability of eosinophilic infiltrates in eosinophilic esophagitis is not well described. This study aimed to determine the distribution of esophageal eosinophilia and the utility of histologic cut-points for eosinophilic esophagitis diagnosis in subjects undergoing endoscopy. We performed a prospective study of adults undergoing outpatient endoscopy. Research protocol esophageal biopsies were obtained from all subjects. Incident cases of eosinophilic esophagitis were diagnosed per consensus guidelines. Biopsies were interpreted following a validated protocol, and maximum eosinophil counts (eosinophils per high-power field; eos/hpf) were determined. Histologic analyses were performed on a per-patient, per-biopsy, and per-hpf basis. There were 213 patients, yielding 923 esophageal biopsies with 4588 hpfs. Overall, 48 patients (23\%), 165 biopsy fragments (18\%), and 449 hpfs $(10 \%)$ had $\geq 15$ eos/hpf; most subjects had no or low levels of eosinophils. In the eosinophilic esophagitis cases, 119 biopsy fragments (63\%) and 332 hpfs (36\%) had $\geq 15$ eos/hpf. There was a mean 104-fold difference between the lowest and highest hpf eosinophil count for the eosinophilic esophagitis patients; $85 \%$ of the biopsies from eosinophilic esophagitis cases also had at least one hpf with $<15$ eos/hpf. The cut-point of 15 eos/hpf had a sensitivity of $100 \%$ and a specificity of $96 \%$ for diagnosis of eosinophilic esophagitis. In conclusion, most patients have little to no esophageal eosinophilia. In patients with eosinophilic esophagitis, there was marked variability in the eosinophil counts by biopsy and by hpf within a given biopsy. Additionally, the 15 eos/hpf cut-point was highly sensitive and specific for eosinophilic esophagitis. Multiple esophageal biopsies from different locations should be obtained to optimize eosinophilic esophagitis diagnosis.

Modern Pathology (2015) 28, 383-390; doi:10.1038/modpathol.2014.110; published online 12 September 2014

The human esophagus is lined with a stratified squamous epithelium that is devoid of eosinophils under normal conditions. The presence of esophageal eosinophilia has been increasingly reported over the last two decades. ${ }^{1-5}$ While the majority of recent reports focus on eosinophilic esophagitis as the cause, esophageal eosinophilia itself is nonspecific and has a wide differential diagnosis. ${ }^{6-9}$ In

Correspondence: Dr ES Dellon MD, MPH, Medicine, Division of Gastroenterology and Hepatology, University of North Carolina School of Medicine, CB\#7080, Bioinformatics Building, 130 Mason Farm Road, Chapel Hill, NC 27599-7080, USA.

E-mail: edellon@med.unc.edu

Received 29 April 2014; revised 22 June 2014; accepted 23 June 2014; published online 12 September 2014 addition to eosinophilic esophagitis, the most common causes include gastroesophageal reflux disease and proton pump inhibitor-responsive esophageal eosinophilia, a condition where esophageal eosinophilia may not be attributed to reflux and resolves after proton pump inhibitor treatment. ${ }^{10-13}$ Additionally, pill esophagitis, drug reactions, infections, Crohn's disease, autoimmune and connective tissue disorders, achalasia, graft-vshost disease, and other conditions have also been associated with esophageal eosinophilia. 6,7

The spectrum of esophageal eosinophilia in patients presenting for upper endoscopy and esophageal biopsy is not well described. In eosinophilic esophagitis in particular, a level of 15 eosinophils per high-power field (eos/hpf) has been adopted as 
the histologic cut-point in diagnostic algorithms, ${ }^{4,6,7}$ but this value is largely empiric and its appropriateness has never been rigorously tested. ${ }^{14}$ Further, the eosinophilic infiltrate can be patchy and unevenly distributed in eosinophilic esophagitis, but the full degree of variability is not well described. ${ }^{8,9}$ These poorly defined parameters impact clinical guidelines, biopsy protocols, and histologic analysis.

The aims of this study were to determine the distribution of esophageal eosinophilia in a wide range of subjects undergoing endoscopy, describe variability of eosinophil counts in patients with eosinophilic esophagitis at the per-patient, perbiopsy, and per-hpf level, and assess the utility of the 15 eos/hpf cut-point.

\section{Materials and methods}

\section{Patients and Esophageal Biopsy Sources}

This was a prospective study at the University of North Carolina from 2009 to 2012. We enrolled consecutive adults from age 18 to 80 years undergoing outpatient esophagogastroduodenoscopy who had either dysphagia or symptoms of gastroesophageal reflux disease. Patients were excluded if they had a known eosinophilic gastrointestinal disorder, including eosinophilic esophagitis, were anticoagulated or having an active GI bleed, had known esophageal varices, esophageal cancer, or prior esophageal surgery, had medical instability precluding enrollment, or were unable to read or understand the consent form. Details of this study design have been previously reported. ${ }^{13}$

Cases of eosinophilic esophagitis were defined as per consensus guidelines. ${ }^{6,7}$ Specifically, patients were required to have at least one typical symptom of esophageal dysfunction (dysphagia, food impaction, or heartburn); at least 15 eos/hpf on esophageal biopsy after an 8-week proton pump inhibitor trial (20-40 mg twice daily of any of the available agents, selected at the discretion of the clinician); and other causes of esophageal eosinophilia excluded. ${ }^{6,7}$ Subjects without eosinophilic esophagitis did not meet these clinical or histologic criteria, but could have other findings or diagnoses. Of note, a noneosinophilic esophagitis subject could have $\geq 15$ eos/hpf attributable to a different cause, such as proton pump inhibitor-responsive esophageal eosinophilia, reflux, achalasia or esophageal dysmotility, and infection. ${ }^{7}$ Samples from the proton pump inhibitor-responsive esophageal eosinophilia patients were analyzed after the proton pump inhibitor trial, and were included in the group of subjects without eosinophilic esophagitis; all of these proton pump inhibitor-responsive esophageal eosinophilia subjects were therefore on proton pump inhibitor. All eosinophilic esophagitis subjects were also on proton pump inhibitor, as per diagnostic guidelines. The other study subjects without eosinophilic esophagitis could be on proton pump inhibitor as clinically indicated at the discretion of their referring provider.

During the endoscopy, a total of five research protocol esophageal biopsies were obtained (two from the proximal, one from the mid, and two from the distal esophagus) to maximize eosinophilic esophagitis diagnostic sensitivity. ${ }^{15}$ Distal biopsies were obtained $3 \mathrm{~cm}$ above the gastroesophageal junction, mid esophageal biopsies were obtained $10 \mathrm{~cm}$ above the gastroesophageal junction, and proximal esophageal biopsies were obtained $15 \mathrm{~cm}$ above the gastroesophageal junction. Each esophageal biopsy fragment was collected, labelled, processed, and embedded into paraffin separately. Concomitant gastric and duodenal biopsies were used to exclude eosinophilic gastritis or gastroenteritis. At the discretion of the endoscopist, additional clinical biopsies could be taken as needed. The study was approved by the University of North Carolina Institutional Review Board, and subjects provided informed consent before endoscopy.

\section{Histologic Analysis}

The study pathologists utilized our previously validated protocol to determine eosinophil counts and associated findings for each of the biopsy fragments obtained. ${ }^{16,17}$ In brief, slides were deidentified without reference to clinical data or endoscopic findings and then scanned, digitized, and viewed with Aperio ImageScope (Aperio Technologies, Vista, CA, USA). For each of the five biopsy fragments, five microscopy fields were assessed to determine the maximum eosinophil density (eosinophils $/ \mathrm{mm}^{2}$ (eos $\left./ \mathrm{mm}^{2}\right)$ ). For reporting and to compare the eosinophil densities with results from other studies, they were converted to eosinophil counts (eos/hpf) for an hpf size of $0.24 \mathrm{~mm}^{2}$, the size of an average field as reported in the literature. ${ }^{14}$

The eosinophil infiltration was further examined to determine whether it was patchy (defined as localized eosinophilia $\geq 15$ eos/hpf in only one hpf in the biopsy) or diffuse (eosinophilic inflammation seen in multiple hpfs) throughout the entire biopsy sample, as well as whether the eosinophil distribution throughout the mucosa was superficial only, basal only, or diffuse (throughout the epithelium). Additionally, the presence of eosinophilic microabscesses (clusters of $\geq 4$ eosinophils), eosinophil degranulation, basal layer hyperplasia (when evaluable in properly oriented specimens), spongiosis, and lamina propria fibrosis (if adequate subepithelial stroma was present) were recorded as defined in previous publications. ${ }^{8,9}$

\section{Statistical Analysis}

Descriptive statistics were used to summarize characteristics of the study population. Histologic 
findings were analyzed on a per-patient, per-biopsy, and per-hpf basis. For the per-patient analysis, the maximum eosinophil count found in any specimen from that patient was the outcome of interest; for the per-biopsy analysis, the maximum eosinophil count from any of the hpfs within that biopsy fragment was reported; and for the per-hpf analysis, data from every hpf were analyzed separately. Histograms representing the distributions of eosinophil counts for each of these analyses, both overall and by location of the biopsy fragments (distal, mid, or proximal esophagus) were constructed. The hpf analysis was also used to determine patchiness of the eosinophilic infiltrate. Specifically, the variation in level of esophageal eosinophilia was calculated as the fold-change between the minimum and maximum eosinophil count in each set of biopsies. Finally, we calculated sensitivity, specificity, positive predictive value, and negative predictive value of using 15 eos/hpf as the histologic cut-point for eosinophilic esophagitis. We also made the same calculations for cut-points of 10 and 20 eos/hpf. Statistical analysis was performed using Stata version 9 (Statacorp, College Station, TX, USA).

\section{Results}

Esophageal biopsies were analyzed from 213 patients. The mean age was 49 years (range 18-79), $48 \%$ were male, and $81 \%$ were white (Table 1 ). As expected with the study design, dysphagia was the most common symptom (77\%) and gastroesophageal reflux disease was the most common diagnosis (21\%). eosinophilic esophagitis was found in 41 patients $(19 \%)$, proton pump inhibitor-responsive esophageal eosinophilia was seen in $24(11 \%)$, and achalasia or other motility disorders were seen in 25 (11\%). These 213 patients yielded a total of 923 biopsy fragments containing $4588 \mathrm{hpfs}$ that were evaluated in the histologic analysis.

\section{Spectrum of Esophageal Eosinophilia in all Subjects Undergoing Endoscopy}

The majority of subjects had low levels or no esophageal eosinophilia noted on per-patient, perbiopsy, and per-hpf analyses (Figure 1). A total of 150 patients $(70 \%)$ had 10 eos/hpf or less, $96(45 \%)$ had 0 eos/hpf, and $48(23 \%)$ had $\geq 15$ eos/hpf. A total of 739 biopsy fragments $(80 \%)$ had 10 or less eos/hpf, $553(60 \%)$ had 0 , and $165(18 \%)$ had $\geq 15$. A total of $4034 \mathrm{hpfs}(88 \%)$ had 10 of less eos/hpf, $3358(73 \%)$ had 0 , and $449(10 \%)$ had $\geq 15$. The overall range of the maximum eosinophil counts was from 0 to $466 \mathrm{eos} / \mathrm{hpf}$.

Among all subjects, the mean of the maximum eosinophil counts on the per-patient, per-biopsy, and per-hpf analyses was 25, 13, and 7 eos/hpf, respectively (Table 2). However, the median values were much lower $(1.5,0$, and 0 eos/hpf, respec-
Table 1 Characteristics of the study population

\begin{tabular}{|c|c|}
\hline & $\begin{array}{l}\text { Total population } \\
\quad(\mathrm{n}=213)\end{array}$ \\
\hline Age (mean \pm s.d.) & $49.2 \pm 15.4$ \\
\hline Male $(n, \%)$ & $102(48)$ \\
\hline White $(n, \%)$ & $173(81)$ \\
\hline \multicolumn{2}{|l|}{ Symptoms/upper endoscopy indication $(n, \%)$} \\
\hline Dysphagia & $165(77)$ \\
\hline Heartburn & 30 (14) \\
\hline Abdominal pain & $23(11)$ \\
\hline Nausea/vomiting & $6(3)$ \\
\hline \multicolumn{2}{|l|}{ Upper endoscopy findings (n, \%) } \\
\hline Normal & $37(17)$ \\
\hline Rings & $74(35)$ \\
\hline Stricture & $47(22)$ \\
\hline Narrowing & $27(13)$ \\
\hline Furrows & $61(29)$ \\
\hline Crêpe-paper & $4(2)$ \\
\hline White plaques/exudates & 35 (16) \\
\hline Decreased vascularity & $17(8)$ \\
\hline Erosive esophagitis & $34(16)$ \\
\hline Schatzki's ring & $17(8)$ \\
\hline Hiatal hernia & $58(27)$ \\
\hline Dilation performed & $68(32)$ \\
\hline $\begin{array}{l}\text { Proton pump inhibitor use at the time of } \\
\text { endoscopy }(n, \%)^{\mathrm{a}}\end{array}$ & $168(79)$ \\
\hline \multicolumn{2}{|l|}{ Diagnoses (n, \%) } \\
\hline Eosinophilic esophagitis & $41(19)$ \\
\hline $\begin{array}{l}\text { Proton pump inhibitor-responsive } \\
\text { esophageal eosinophilia }\end{array}$ & $24(11)$ \\
\hline Control & $148(70)$ \\
\hline Normal & $11(5)$ \\
\hline Reflux & $45(21)$ \\
\hline Peptic stricture & $10(5)$ \\
\hline Non-peptic stricture & $9(4)$ \\
\hline Schatzki's ring & $11(5)$ \\
\hline Achalasia & $9(4)$ \\
\hline Non-achalasia esophageal dysmotility & $16(7)$ \\
\hline Functional esophageal disorders & $6(3)$ \\
\hline Other & $31(11)$ \\
\hline
\end{tabular}

${ }^{\mathrm{a}}$ By definition, all of the eosinophilic esophagitis and proton pump inhibitor-responsive esophageal eosinophilia cases were on a proton pump inhibitor at endoscopy; 103 of the other subjects (70\%) were on a proton pump inhibitor at the discretion of their referring physician at the time of endoscopy.

tively), indicating that the mean values were impacted by the set of patients with very high eosinophil counts. Eosinophil degranulation was observed in $33 \%$ of patients, $24 \%$ of biopsies, and $14 \%$ of hpfs, and microabscesses were seen in 13,7 , and $3 \%$, respectively. In this patient population, subepithelial stroma was not routinely noted, being present in only $38 \%$ of patients and $15 \%$ of all esophageal biopsies. Fibrosis of the lamina propria was also not common, seen in $8 \%$ of patients and $3 \%$ of biopsies. Qualitatively, the eosinophilic distribution was diffuse throughout the mucosa (ie, no superficial vs basal predominance and the inflammation was seen throughout the entire mucosa), but was patchy throughout the entire biopsy specimen (ie, there were some areas with prominent 

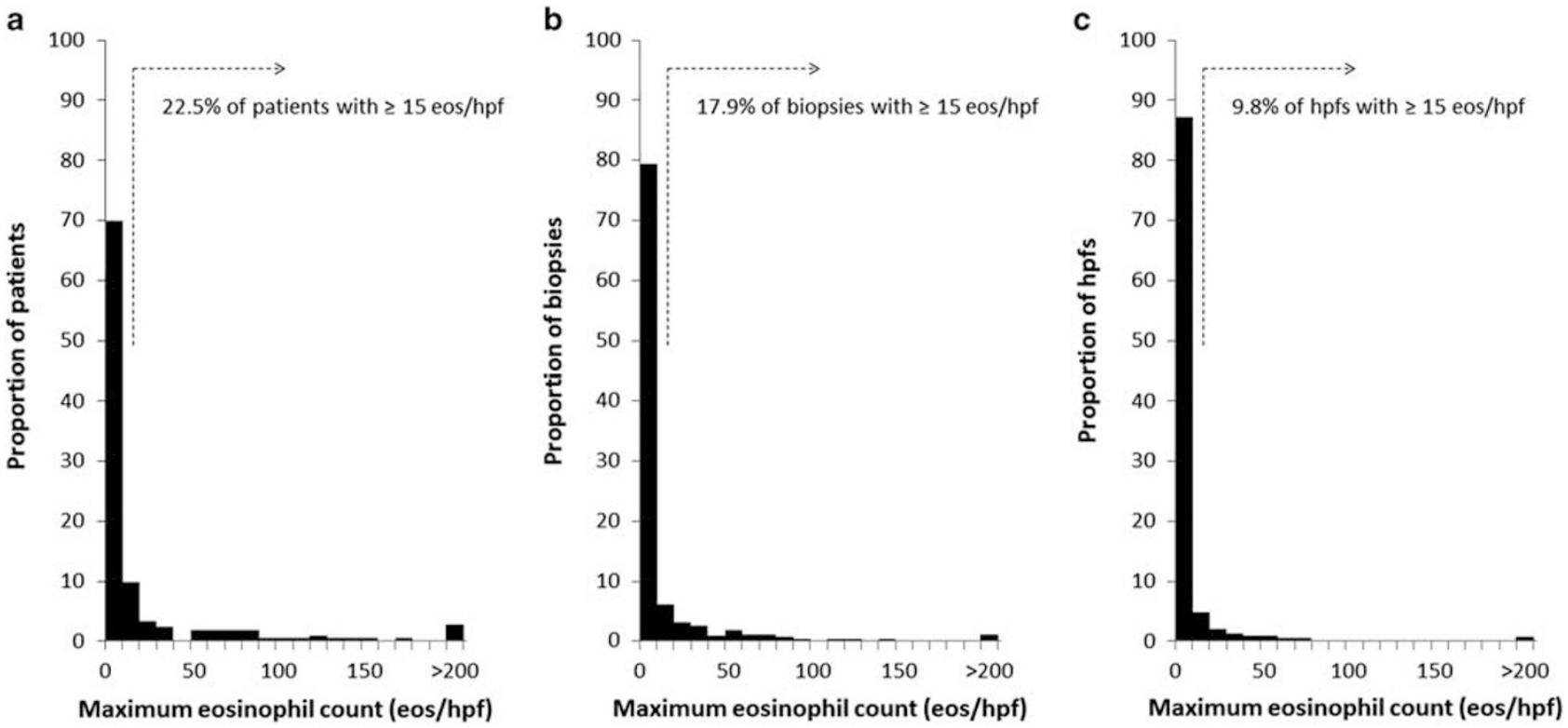

Figure 1 Histograms of the distribution of esophageal eosinophilia (eos/hpf) in patients undergoing upper endoscopy. (a) Histogram for the per-patient analysis. (b) Histogram for the per-biopsy analysis. (c) Histogram for the per-hpf analysis.

Table 2 Histologic features analyzed by patient, by biopsy, and by high-power field

\begin{tabular}{|c|c|c|c|}
\hline & Per patient $(\mathrm{n}=213)$ & Per biopsy $(\mathrm{n}=923)$ & Per hpf $(\mathrm{n}=4588)$ \\
\hline$\geq 15 \mathrm{eos} / \mathrm{hpf}(n, \%)$ & $48(23)$ & $165(18)$ & $449(10)$ \\
\hline Max eosinophil count (mean eos $/ \mathrm{hpf} \pm$ s.d., range) & $24.6 \pm 64.9(0-466)$ & $13.2 \pm 40.9(0-466)$ & $6.6 \pm 25.9(0-466)$ \\
\hline Max eosinophil count (median eos/hpf, IQR) & $1.5(0-14)$ & $0(0-6.2)$ & $0(0-1.4)$ \\
\hline Degranulation $(n, \%)$ & $71(33)$ & $218(24)$ & $645(14)$ \\
\hline Microabscess $(n, \%)$ & 27 (13) & $60(7)$ & $136(3)$ \\
\hline Basal layer evaluable $(n, \%)$ & 212 (99) & $876(95)$ & - \\
\hline Basal hyperplasia $(n, \%)$ & $48(23)$ & $102(12)$ & - \\
\hline $25-50 \%$ high & $25(12)$ & $55(6)$ & \\
\hline $50-75 \%$ high & $23(11)$ & $47(5)$ & \\
\hline Spongiosis $(n, \%)$ & $60(28)$ & $153(17)$ & - \\
\hline Subepithelial stroma present $(n, \%)$ & $81(38)$ & $151(16)$ & - \\
\hline Lamina prop fibrosis $(n, \%)$ & $16(8)$ & $26(3)$ & - \\
\hline Mucosal distribution $(n, \%)^{\mathrm{a}}$ & - & & - \\
\hline Basal & & $39(14)$ & \\
\hline Superficial & & $50(19)$ & \\
\hline Diffuse & & $181(67)$ & \\
\hline Biopsy distribution $(n, \%)^{\mathrm{a}}$ & - & & - \\
\hline Patchy & & $216(67)$ & \\
\hline Diffuse & & $109(33)$ & \\
\hline
\end{tabular}

${ }^{\mathrm{a}}$ Percentages are calculated for those biopsies where there are eosinophils present and the distribution of eosinophils can be assessed.

eosinophilic infiltration, but other areas where this was much less pronounced).

\section{Variability of Esophageal Eosinophilia in Subjects with Eosinophilic Esophagitis}

When analyzing those diagnosed with eosinophilic esophagitis $(n=41)$, despite high eosinophil counts at the patient level (mean of the maximum eosinophil count $=108$ eos $/ \mathrm{hpf}$ ) with frequent associated histologic findings (degranulation in 93\%, microabscesses in $59 \%$, basal layer hyperplasia in $66 \%$, spongiosis in $80 \%$, and lamina propria fibrosis in $27 \%$ ), there was substantial variability in eosinophil counts on per-biopsy and per-hpf analysis (Figure 2). Of the 189 biopsy fragments, $43(23 \%)$ had $\leq 5$ eos/ hpf, and $119(63 \%)$ had $\geq 15$ eos/hpf. Of the 932 hpfs, 445 (48\%) had $\leq 5$ eos/hpf, and 332 (36\%) had $\geq 15$ eos/hpf. There were no substantial differences between eosinophil counts and associated histologic finding on either the per-biopsy or per-hpf analysis based on the location of the sample (Table 3).

With this level of variability, there was a mean 104-fold difference between the lowest and highest eosinophil count for the eosinophilic esophagitis patients. While every patient with eosinophilic esophagitis had at least one hpf with an eosinophil count of $\geq 15$ eos/hpf by definition, $85 \%$ of the 

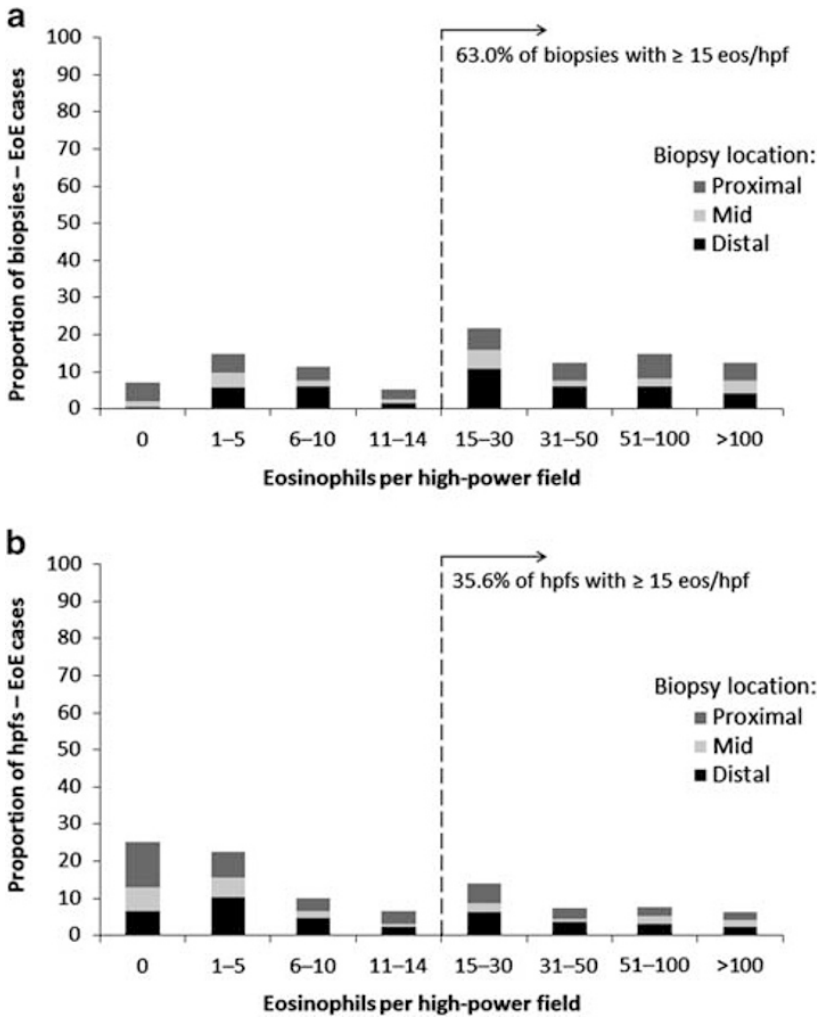

Figure 2 Distribution of esophageal eosinophilia in patients with eosinophilic esophagitis, stratified by biopsy location. (a) Distribution for the per-biopsy analysis. (b) Distribution for the perhpf analysis. The dark gray represents proximal locations, the light gray is mid-esophagus, and the black represents distal locations. biopsies also had at least one hpf with $<15$ eos/hpf. The average of the maximum eosinophil counts for all hpfs was 108 eos/hpf (range: 16-466). The average of the minimum eosinophil counts for all hpfs was 1 eos/hpf (range 0-11).

\section{Operating Characteristics of the Eosinophil Count and Other Histologic Findings}

When the current histologic cut-point of 15 eos/hpf for diagnosis of eosinophilic esophagitis was assessed at the per-patient level, it had a sensitivity of $100 \%(41 / 41)$, a specificity of $96 \%$ (165/172), a positive predictive value of $85 \%(41 / 48)$, and a negative predictive value of $100 \%(165 / 165)$. Alternative cut-points of 10 or $20 \mathrm{eos} / \mathrm{hpf}$ did not perform as well, nor did specific histologic findings (Table 4). Though eosinophilic microabscesses and lamina propria fibrosis were specific for eosinophilic esophagitis (98 and 97\%, respectively), they were not sensitive (56 and $27 \%$, respectively). On a perbiopsy level, the cut-point of 15 eos/hpf performed less well, with a sensitivity of $63 \%$ (119/189), a specificity of $94 \%(688 / 734)$, a positive predictive value of $70 \%(46 / 165)$, and a negative predictive value of $91 \%(688 / 758)$.

\section{Discussion}

With increasing interest in eosinophilic esophagitis in recent years, the finding of eosinophilia on

Table 3 Histologic features of eosinophilic esophagitis cases stratified by esophageal level and analyzed by biopsy and by high-power field

\begin{tabular}{|c|c|c|c|c|c|c|}
\hline & \multicolumn{3}{|c|}{$\begin{array}{l}\text { Per-biopsy } \\
\text { analysis }(\mathrm{n}=189)\end{array}$} & \multicolumn{3}{|c|}{$\begin{array}{c}\text { Per-hpf } \\
\text { analysis }(\mathrm{n}=932)\end{array}$} \\
\hline & $\begin{array}{l}\text { Distal } \\
(\mathrm{n}=75)\end{array}$ & $\begin{array}{c}\text { Mid } \\
(\mathrm{n}=40)\end{array}$ & $\begin{array}{l}\text { Proximal } \\
(\mathrm{n}=74)\end{array}$ & $\begin{array}{c}\text { Distal } \\
(\mathrm{n}=370)\end{array}$ & $\begin{array}{c}\text { Mid } \\
(\mathrm{n}=198)\end{array}$ & $\begin{array}{l}\text { Proximal } \\
(\mathrm{n}=364)\end{array}$ \\
\hline$\geq 15$ eos/hpf $(n, \%)$ & $53(75)$ & $24(60)$ & $42(57)$ & 144 (39) & $65(33)$ & $123(34)$ \\
\hline $\begin{array}{l}\text { Max eosinophil count (mean eos/ } \\
\text { hpf } \pm \text { s.d.) }\end{array}$ & $48.8 \pm 73.3$ & $56.9 \pm 93.4$ & $44.9 \pm 65.5$ & $26.8 \pm 53.6$ & $26.5 \pm 54.4$ & $22.8 \pm 42.9$ \\
\hline Max eosinophil count & 24.1 & 18.5 & 21.1 & 8.7 & 4.1 & 6.7 \\
\hline (median eos/hpf, IQR) & $(9.0-58.2)$ & $(4.5-70.8)$ & $(4.7-57.2)$ & $(2.5-26)$ & $(0-23)$ & $(0-26)$ \\
\hline Degranulation $(n, \%)$ & $57(76)$ & $28(70)$ & $49(66)$ & $194(52)$ & $87(44)$ & $153(42)$ \\
\hline Microabscess $(n, \%)$ & $21(28)$ & $12(30)$ & $25(32)$ & $48(13)$ & $24(12)$ & $57(16)$ \\
\hline Basal layer evaluable (n, \%) & $72(96)$ & $39(98)$ & $70(95)$ & - & - & - \\
\hline Basal hyperplasia ( $n, \%$ ) & & & & - & - & - \\
\hline $25-50 \%$ high & $12(22)$ & $7(22)$ & $9(16)$ & & & \\
\hline 50-75\% high & $13(24)$ & $8(25)$ & $12(21)$ & & & \\
\hline Spongiosis $(n, \%)$ & $43(57)$ & $20(51)$ & $33(45)$ & - & - & - \\
\hline Subepithelial stroma present $(n, \%)$ & $23(31)$ & $13(33)$ & $21(28)$ & - & - & - \\
\hline Lamina prop fibrosis $(n, \%)$ & $7(9)$ & $5(13)$ & 8 (11) & - & - & - \\
\hline Mucosal distribution $(n, \%)^{\mathrm{a}}$ & & & & - & - & - \\
\hline Basal & $3(5)$ & $2(6)$ & $1(2)$ & & & \\
\hline Superficial & $14(23)$ & $5(16)$ & $13(24)$ & & & \\
\hline Diffuse & $44(72)$ & $25(78)$ & $40(74)$ & & & \\
\hline Biopsy distribution $(n, \%)^{\mathrm{a}}$ & & & & - & - & - \\
\hline Patchy & $39(55)$ & $23(64)$ & $31(50)$ & & & \\
\hline Diffuse & $23(47)$ & $13(36)$ & $31(50)$ & & & \\
\hline
\end{tabular}

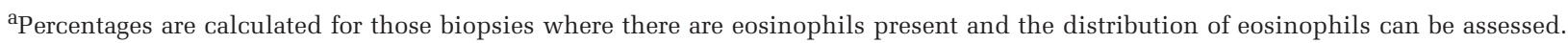


Table 4 Operating characteristics of the eosinophil counts and histologic features for diagnosis of eosinophilic esophagitis

\begin{tabular}{|c|c|c|c|c|c|c|}
\hline & $\geq 15$ eos/hpf & $\geq 10 \mathrm{eos} / \mathrm{hpf}$ & $\geq 20 \mathrm{eos} / \mathrm{hpf}$ & Degranulation & Microabscesses & $\begin{array}{c}\text { Lamina } \\
\text { propria fibrosis }\end{array}$ \\
\hline Sensitivity & 100 & 100 & 90 & 93 & 56 & 27 \\
\hline Specificity & $96^{\mathrm{a}}$ & 87 & 97 & 81 & 98 & 97 \\
\hline Positive predictive value & 85 & 35 & 86 & 54 & 89 & 69 \\
\hline Negative predictive value & 100 & 100 & 98 & 98 & 91 & 85 \\
\hline
\end{tabular}

${ }^{a}$ Of the seven patients who had $\geq 15$ eos/hpf not attributable to either eosinophilic esophagitis or proton pump inhibitor-responsive esophageal eosinophilia, reflux was the cause in six, and esophageal dysmotility leading to stasis esophagitis was the cause in one.

esophageal biopsy is being more frequently encountered. However, the spectrum of esophageal eosinophilia has not previously been well described and the variability in this finding had yet to be fully quantified. In this study of prospectively obtained esophageal biopsy specimens, histologic findings related to esophageal eosinophilia were analyzed to determine the distribution and variability of esophageal eosinophilia in subjects undergoing endoscopy.

Our study had several key findings. First, the large majority of patients undergoing upper endoscopy had little to no esophageal eosinophilia regardless of whether the analysis was at the per-patient, perbiopsy, or per-hpf level. This solidifies the concept that having an eosinophilic infiltrate in the esophagus is abnormal. Second, patients with eosinophilic esophagitis not only had high levels of esophageal eosinophilia, but also had marked variability in the eosinophil counts on both the per-biopsy and perhpf level. This implies that multiple biopsies from multiple locations need to be obtained, and that multiple hpfs must be examined on each biopsy. Finally, we provide quantifiable prospective data about the distribution of eosinophils in eosinophilic esophagitis cases compared with subjects without eosinophilic esophagitis, and provide the first evidence-based support for the $15 \mathrm{eos} / \mathrm{hpf}$ cut-point for diagnosis of eosinophilic esophagitis.

The variability of the eosinophilic infiltrate in eosinophilic esophagitis has been described before, but these descriptions have largely been limited to cohorts of eosinophilic esophagitis patients, either in subanalyses from clinical trials ${ }^{18-21}$ or in retrospective studies, ${ }^{15,22}$ without a non-eosinophilic esophagitis comparator group for context. In these latter two studies, the authors determined that obtaining a total of five biopsies yielded a diagnostic sensitivity of $100 \%$ for eosinophilic esophagitis with a histologic cut-point of 15 eos/hpf. ${ }^{15,22}$ A unique analysis of an esophagectomy specimen from a patient with eosinophilic esophagitis was able to explicitly show the variability in eosinophilia. ${ }^{23}$ This study found that just $31.5 \%$ of hpfs (a total of 1215 were examined from this single patient) had an eosinophil count of $\geq 15$ eos/hpf, a finding that is very similar to what we present here for our entire patient population on the per-hpf analysis. The same group has also presented preliminary data on six patients undergoing a strict and extensive esophageal biopsy protocol. ${ }^{24}$ This suggested that up to 17 biopsies might be required to find high levels of esophageal eosinophilia, with fewer in areas with endoscopic findings such as white plaques and more from areas of normalappearing esophageal mucosa. An additional retrospective study of an eosinophilic esophagitis cohort performed a per-biopsy analysis of 1342 fragments and concluded that obtaining at least 4 biopsy specimens would provide a diagnostic yield of $>99 \%$ with a 15 eos/hpf cut-point. ${ }^{25}$ Our biopsy protocol obtained random biopsies from the distal, mid, and proximal esophagus, and while there was a large variation in eosinophil counts between hpfs within biopsies, for the eosinophilic esophagitis cases $63 \%$ of biopsies had at least $1 \mathrm{hpf}$ with $\geq 15$ eos/hpf. This result is consistent with these prior studies and lends support to the current guidelines recommendation of obtaining 2-4 biopsies from at least 2 locations in the esophagus to obtain adequate tissue for eosinophilic esophagitis diagnosis.

Because these prior studies are nested within eosinophilic esophagitis cohorts, they are unable to assess the utility of the level of 15 eos/hpf as a diagnostic cut-point. This cut-point was originally recommended in the first iteration of the eosinophilic esophagitis clinical guidelines ${ }^{4}$ to attempt to bring a measure of uniformity to variable case definitions in the literature. ${ }^{14}$ While based on data showing that that lower levels of eosinophilic infiltration in the esophagus were often due to gastroesophageal reflux disease, ${ }^{26,27}$ there has been little empiric data to support this recommendation. ${ }^{28,29}$ Our study is the first of which we are aware to prospectively enroll patients undergoing endoscopy and review biopsies from both cases of eosinophilic esophagitis and subjects without eosinophilic esophagitis to determine the utility of the 15 eos/hpf cut-point. We found that this cutpoint has an excellent sensitivity, specificity, and negative predictive value for diagnosis of eosinophilic esophagitis, and performs better than alternative cut-points such as 10 or 20 eos/hpf. 
These findings also support current diagnostic recommendations. ${ }^{6,7}$

It is important to acknowledge the limitations and strengths of this study. First, it is a single center study at an esophageal referral center, but the characteristics of the eosinophilic esophagitis cases are similar to those reported in multiple other studies. ${ }^{15,30-33}$ Second, this study only enrolled adults, so it is unknown whether the current results would be applicable to children. Third, it is important to note that the eosinophil counts presented in this study for subjects with eosinophilic esophagitis and proton pump inhibitor-responsive esophageal eosinophilia were measured after therapy with a proton pump inhibitor, so while patients with proton pump inhibitor-responsive esophageal eosinophilia are included, their samples are categorized with the non-eosinophilic esophagitis cases. We are therefore unable to comment on variability in eosinophil counts in patients with proton pump inhibitor-responsive esophageal eosinophilia before the proton pump inhibitor trial. We feel that the strengths of this study, including its prospective design, comprehensive and standardized histopathologic analysis using a previously validated and reproducible protocol, and ability to compare eosinophilic esophagitis cases with non- eosinophilic esophagitis controls, outweigh the potential limitations.

In conclusion, this study of the spectrum of esophageal eosinophilia in patients undergoing upper endoscopy and biopsy found that most patients have little to no esophageal eosinophilia, but the levels of esophageal eosinophilia in patients with eosinophilic esophagitis vary tremendously on per-patient, per-biopsy, and per-hpf analyses. Because nearly two-thirds of biopsies had at least 15 eos/hpf, obtaining at least 3 biopsies may provide adequate diagnostic yield, but the marked variability implies that when more biopsies are obtained, the sensitivity of diagnosis increases. Because approximately only one-third of hpfs had at least 15 eos/hpf, examining multiple hpfs perbiopsy specimen is also important. In addition, these data support the current histologic cut-point of 15 eos/hpf for diagnosis of eosinophilic esophagitis. Data from this study can be used to make biopsyrelated recommendations in future iterations of eosinophilic esophagitis guidelines, and may be helpful for biopsy acquisition and histologic assessment protocols in clinic trial design.

\section{Acknowledgments}

This study was conducted with support, in part, by NIH K23 DK090073. It also utilized the Histology Core of the University of North Carolina Center for Gastrointestinal Biology and Disease (NIH P30DK034987) and the University of North Carolina Translational Pathology Lab (NIH P30CA016086).

\section{Disclosure/conflict of interest}

The authors declare no conflict of interest.

\section{References}

1 Attwood SE, Smyrk TC, Demeester TR, et al. Esophageal eosinophilia with dysphagia: a distinct clinicopathologic syndrome. Dig Dis Sci 1993;38:109-116.

2 Straumann A, Spichtin HP, Bernoulli R, et al. [Idiopathic eosinophilic esophagitis: a frequently overlooked disease with typical clinical aspects and discrete endoscopic findings]. Schweiz Med Wochenschr 1994;124:1419-1429.

3 Kelly KJ, Lazenby AJ, Rowe PC, et al. Eosinophilic esophagitis attributed to gastroesophageal reflux: improvement with an amino acid-based formula. Gastroenterology 1995;109:1503-1512.

4 Furuta GT, Liacouras CA, Collins $\mathrm{MH}$, et al. Eosinophilic esophagitis in children and adults: a systematic review and consensus recommendations for diagnosis and treatment. Gastroenterology 2007;133: 1342-1363.

5 Katzka DA. Eosinophilic esophagitis: from rookie of the year to household name. Clin Gastroenterol Hepatol 2009; 7:370-371.

6 Liacouras CA, Furuta GT, Hirano I, et al. Eosinophilic esophagitis: updated consensus recommendations for children and adults. J Allergy Clin Immunol 2011; 128:e6.

7 Dellon ES, Gonsalves N, Hirano I, et al. ACG clinical guideline: evidence based approach to the diagnosis and management of esophageal eosinophilia and eosinophilic esophagitis. Am J Gastroenterol 2013;108: 679-692.

8 Collins $\mathrm{MH}$. Histopathologic features of eosinophilic esophagitis. Gastrointest Endosc Clin N Am 2008;18: 59-71; viii-ix

9 Odze RD. Pathology of eosinophilic esophagitis: what the clinician needs to know. Am J Gastroenterol 2009; 104:485-490.

10 Spechler SJ, Genta RM, Souza RF. Thoughts on the complex relationship between gastroesophageal reflux disease and eosinophilic esophagitis. Am J Gastroenterol 2007;102:1301-1306.

11 Rodrigo S, Abboud G, Oh D, et al. High intraepithelial eosinophil counts in esophageal squamous epithelium are not specific for eosinophilic esophagitis in adults. Am J Gastroenterol 2008;103:435-442.

12 Molina-Infante J, Ferrando-Lamana L, Ripoll C, et al. Esophageal eosinophilic infiltration responds to proton pump inhibition in most adults. Clin Gastroenterol Hepatol 2011;9:110-117.

13 Dellon ES, Speck O, Woodward K, et al. Clinical and endoscopic characteristics do not reliably differentiate PPI-responsive esophageal eosinophilia and eosinophilic esophagitis in patients undergoing upper endoscopy: a prospective cohort study. Am J Gastroenterol 2013;108:1854-1860.

14 Dellon ES, Aderoju A, Woosley JT, et al. Variability in diagnostic criteria for eosinophilic esophagitis: a systematic review. Am J Gastroenterol 2007;102:2300-2313.

15 Gonsalves N, Policarpio-Nicolas M, Zhang Q, et al. Histopathologic variability and endoscopic correlates in adults with eosinophilic esophagitis. Gastrointest Endosc 2006;64:313-319. 
16 Dellon ES, Fritchie KJ, Rubinas TC, et al. Inter- and intraobserver reliability and validation of a new method for determination of eosinophil counts in patients with esophageal eosinophilia. Dig Dis Sci 2010;55:1940-1949.

17 Speck O, Woodward K, Covey S, et al. A training curriculum for pathologists yields highly reproducible esophageal eosinophil counts. Gastroenterology 2013; 144(Suppl 1):S499 (Su 1881).

18 Konikoff MR, Noel RJ, Blanchard C, et al. A randomized, double-blind, placebo-controlled trial of fluticasone propionate for pediatric eosinophilic esophagitis. Gastroenterology 2006;131:1381-1391.

19 Schaefer ET, Fitzgerald JF, Molleston JP, et al. Comparison of oral prednisone and topical fluticasone in the treatment of eosinophilic esophagitis: a randomized trial in children. Clin Gastroenterol Hepatol 2008;6: 165-173.

20 Collins $\mathrm{MH}$, Irani AM, Ngo PD, et al. Reduction of esophageal epithelial pathology and endoscopic abnormalities following treatment with oral budesonide suspension (OBS) in pediatric subjects with eosinophilic esophagitis (EoE). Gastroenterology 2011;140 (Suppl 1):S237 (AB Sa1148).

21 Dellon ES, Speck O, Woodward K, et al. The patchy nature of esophageal eosinophilia in eosinophilic esophagitis: insights from pathology samples from a clinical trial. Gastroenterology 2012;142(Suppl 2):Ab Su1129.

22 Shah A, Kagalwalla AF, Gonsalves N, et al. Histopathologic variability in children with eosinophilic esophagitis. Am J Gastroenterol 2009;104:716-721.

23 Saffari H, Peterson KA, Fang JC, et al. Patchy eosinophil distributions in an esophagectomy specimen from a patient with eosinophilic esophagitis: implications for endoscopic biopsy. J Allergy Clin Immunol 2012;130:798-800.
24 Saffari H, Salek J, Clayton F, et al. Phenotypically differentiated biopsy selection improves EoE detection. Gastroenterology 2012;142(Suppl 1):S430 AB Su1121.

25 Nielsen JA, Lager DJ, Lewin M, et al. The optimal number of biopsy fragments to establish a morphologic diagnosis of eosinophilic esophagitis. Am J Gastroenterol 2014;109:515-520.

26 Winter HS, Madara JL, Stafford RJ, et al. Intraepithelial eosinophils: a new diagnostic criterion for reflux esophagitis. Gastroenterology 1982;83:818-823.

27 Ruchelli E, Wenner W, Voytek T, et al. Severity of esophageal eosinophilia predicts response to conventional gastroesophageal reflux therapy. Pediatr Dev Pathol 1999;2:15-18.

28 Genta RM, Spechler SJ, Souza RF. The twentieth eosinophil. Adv Anat Pathol 2007;14:340-343.

29 Hurrell JM, Genta RM, Melton SD. Histopathologic diagnosis of eosinophilic conditions in the gastrointestinal tract. Adv Anat Pathol 2011;18: 335-348.

30 Straumann A, Spichtin HP, Grize L, et al. Natural history of primary eosinophilic esophagitis: a follow-up of 30 adult patients for up to 11.5 years. Gastroenterology 2003;125:1660-1669.

31 Prasad GA, Talley NJ, Romero Y, et al. Prevalence and predictive factors of eosinophilic esophagitis in patients presenting with dysphagia: a prospective study. Am J Gastroenterol 2007;102:2627-2632.

32 Veerappan GR, Perry JL, Duncan TJ, et al. prevalence of eosinophilic esophagitis in an adult population undergoing upper endoscopy: a prospective study. Clin Gastroenterol Hepatol 2009;7:420-426.

33 Spergel JM, Brown-Whitehorn TF, Beausoleil JL, et al. 14 years of eosinophilic esophagitis: clinical features and prognosis. J Pediatr Gastroenterol Nutr 2009;48: 30-36. 